\title{
Paresia do nervo oculomotor: uma entidade por vezes difícil de gerir em medicina geral e familiar
}

Ana Martins Ferreira dos Santos ${ }^{1}$

\section{RESUMO}

Introdução: A paresia do nervo oculomotor constitui uma patologia rara na população geral, que acomete principalmente pessoas acima dos 60 anos. Pode manifestar-se por dor ocular, diplopia, ptose e estrabismo, sendo a hipertensão e a diabetes as suas principais etiologias. As mononeuropatias cranianas atingem 3\% da população diabética. Poderá ser a manifestação de situações potencialmente fatais, como aneurismas cerebrais.

Descrição do caso: Mulher de 55 anos, bom estado geral, embora com várias comorbilidades importantes, incluindo diabetes mellitus tipo 1 com mau controlo metabólico. A 12/05/2019 refere início súbito de dor ocular e supraciliar direitas, com os seguintes sintomas associados, também a nível do olho direito: visão turva, diplopia, fotofobia e estrabismo divergente de novo. Após dois contactos prévios com instituições de saúde por este motivo, acabou por ser referenciada ao serviço de urgência pela sua médica de família, quatro dias após início do quadro. Foi-lhe diagnosticada paresia do III par craniano direito, tendo-se adotado uma atitude expectante. A remissão espontânea das queixas ocorreu após oito semanas.

Comentário: Pretende-se com o relato deste caso alertar para a importância do médico de família na continuidade e articulação de cuidados, pois sendo este o médico assistente por excelência do seu utente será aquele que mais o conhece e que melhor poderá valorizar alterações importantes que possam surgir de novo. Por outro lado, tem por objetivo realçar a importância da rápida atuação perante a sua suspeita clínica, bem como do controlo dos fatores de risco cardiovasculares na população diabética, revelando-se importante do ponto de vista formativo para a prática clínica do médico de família.

Palavras-chave: Doença do nervo oculomotor; Nervo motor ocular comum; Diabetes mellitus.

\section{INTRODUÇÃO}

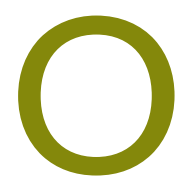

III par craniano, também conhecido como nervo oculomotor, é formado por dois tipos de fibras nervosas:

- Parassimpáticas (externas). Inervam dois músculos intrínsecos do olho: o ciliar e esfíncter da pupila;

- Simpáticas (internas). Inervam músculos extrínsecos do olho, o elevador da pálpebra superior e quatro músculos extraoculares: reto superior, reto interno, reto inferior e pequeno oblíquo).

Juntamente com os nervos troclear (IV par, que inerva o reto lateral) e abducente (VI par, que inerva o

1. Médica especialista. UCSP Oliveira do Hospital, ACeS Pinhal Interior Norte, Oliveira do Hospital. oblíquo superior), o III par é responsável pela inervação dos seis músculos extraoculares que permitem os movimentos oculares. ${ }^{1-3}$

\section{Etiologia}

As causas mais frequentes são: isquemia microvascular dos vasa nervosa (25\% dos casos; sendo as principais a diabetes e hipertensão), aneurismas (17\%), traumatismo crânio-encefálico (13\%) e neoplasias (11\%). As restantes causas correspondem a $14 \%$ dos casos, sendo que a etiologia é desconhecida em $20 \%$ dos doentes. $^{2}$

\section{Epidemiologia}

A paresia do III par constitui uma patologia rara 
(quatro casos por 100.000 pessoas). A incidência é igual nos dois sexos. A faixa etária mais afetada é aquela acima dos 60 anos.

\section{Clínica}

- Ptose ( $86 \%$ dos casos): decorrente da paralisia do elevador da pálpebra superior.

- Infraducção e abdução ocular: por ação do reto lateral e do superior oblíquo, que permanecem intactos.

- Diplopia: secundária ao desvio ínfero-externo do olho afetado, levando a que a imagem incida num ponto extrafoveal; contudo, a ptose pode funcionar como uma barreira visual e impedir que o doente se aperceba da visão dupla.

- Pupila fixa e midriática: devido ao atingimento do músculo ciliar e do esfíncter pupilar, respetivamente; ocorre nas paresias completas.

- Dor ocular/palpebral: pode ocorrer nas paresias de causa isquémica, de forma intensa.

As lesões que afetam o nervo oculomotor podem ser uni ou bilaterais (mais raras), isoladas ou complexas (quando envolvem outros nervos cranianos). No caso das lesões isoladas, estas podem ser completas ou incompletas, consoante o tipo de fibras nervosas atingidas, de acordo com a sua localização:

- Paresias totais/completas (43\% dos casos): há envolvimento da musculatura intrínseca e extrínseca (ou seja, das fibras parassimpáticas e simpáticas, respetivamente) e o reflexo pupilar não é preservado. Ocorre porque as fibras parassimpáticas, mais periféricas, são logo afetadas quando ocorre uma compressão externa, como nos aneurismas da artéria comunicante posterior, tumores cerebrais ou hérnias cerebrais internas.

- Paresias parciais/incompletas (57\% dos casos): com atingimento apenas das fibras simpáticas mais internas, afetando só os músculos extrínsecos do olho, poupando assim a função pupilar e de acomodação. Acontece nas lesões microisquémicas (diabetes, hipertensão, aterosclerose cerebral, arterite temporal e miastenia gravis). ${ }^{1-2,4}$

\section{Avaliação complementar}

- Exames imagiológicos. Perante uma situação aguda de paresia do oculomotor é mandatória a rápida rea-

\section{TABELA 1. Principais diagnósticos diferenciais ${ }^{2}$}

Enxaqueca oftalmoplégica

Oftalmoplegia internuclear

Blefaroptose congénita ou adquirida

Anisocoria

Miastenia gravis

Oftalmopatia tiroideia

Arterite de células gigantes

lização de exames de imagem arteriográficos: angiografia por tomografia computadorizada (angioTC), angiografia por ressonância magnética (angioRM) ou angiografia de subtração digital, podendo ser necessária a realização de mais do que um exame nos casos mais complexos.

- Exames laboratoriais. Perante uma suspeita de etiologia vascular deverão ser avaliados os fatores de risco cerebrovasculares, com a medição da tensão arterial e estudo analítico: hemograma, glicemia em jejum, HbAlc e VS. Nos idosos será pertinente excluir arterite de células gigantes, com PCR, VS e biópsia da artéria temporal.

De referir que a presença de fatores de risco vasculares suporta, mas não confirma, uma etiologia isquémica, uma vez que estes são muito frequentes na população geral, mas pode ser outra a causa da paresia do nervo oculomotor. ${ }^{2}$

\section{Tratamento}

- Médico. Indicado nas paresias agudas e nos doentes com mais de 50 anos com antecedentes de diabetes ou hipertensão. Na maioria dos casos opta-se por uma atitude expectante, com tratamento sintomático da dor e diplopia, uma vez que não existe terapêutica médica específica que altere a história natural da doença. Os anti-inflamatórios (ibuprofeno) são os analgésicos de primeira linha. Relativamente à diplopia, quando o ângulo de divergência em relação ao eixo visual é grande poderá ocluir-se temporariamente o olho afetado com um penso ou lente de contacto opaca. Quando o desvio é menor opta-se por aplicar um prisma de Fresnel vertical e/ou 
TABELA 2. Antecedentes patológicos

\begin{tabular}{l} 
Médicos \\
\hline 2019 - Incontinência urinária mista (nova referenciação a \\
consulta de urologia) \\
\hline \\
\hline 2018 - Obstipação \\
\hline 2017 - Litíase vesicular sintomática (cólica biliar), aguardando \\
cirurgia (nova referenciação a consulta de cirurgia geral) \\
\hline 2015 - Insuficiência cardíaca com FEVE diminuída de causa \\
isquémica, portadora de CDI (nova referenciação a consulta de \\
cardiologia) \\
\hline $27 / 05 / 2015$ - Quedas frequentes, com fratura maleolar direita \\
(tratamento conservador com bota gessada) \\
\hline $30 / 01 / 2014$ - Ex-fumadora (30 UMA; um maço/dia, 20-50 anos) \\
\hline 2008 - Depressão, com três episódios de tentativa de suicídio \\
no passado (referenciação consulta de psicologia no Centro \\
de Saúde) \\
\hline 2003 - Dislipidemia mista \\
\hline 1996 - Tiroidite autoimune linfocítica crónica, cursando com \\
hipotiroidismo \\
\hline 1982 - Epilepsia (seguimento em consulta de neurologia)
\end{tabular}

horizontal nas lentes dos óculos. Também podem ser administradas injeções de toxina botulínica no reto lateral, prevenindo assim o desvio lateral do olhar. $^{1-2}$

- Cirúrgico. Aconselha-se nas ptoses ou diplopia que não melhoram em 6-12 meses. O objetivo da cirurgia do estrabismo é permitir o alinhamento do olhar em posição primária, assim como proporcionar uma visão única binocular, o que deverá ocorrer antes da correção da ptose palpebral para prevenir a diplopia. Está também indicado o tratamento neurocirúrgico dos aneurismas saculares. ${ }^{1-2}$

\section{Prognóstico}

A maioria das paresias incompletas melhora parcialmente em quatro semanas e recupera totalmente no intervalo de três meses. Nos casos em que não se veri-

\section{Cirúrgicos}

04 e 27/07/2010 - Cirurgia bilateral a cataratas

25/07/1999 - Cirurgia a síndroma do túnel cárpico à direita, com agravamento severo de dor neuropática e impotência funcional após a cirurgia (referenciação a consulta de ortopedia) fica uma recuperação total dentro de 12-16 semanas deverá ser colocada outra hipótese diagnóstica.

Nos casos secundários, a outra patologia de base (aneurismas saculares, infiltração meníngea infeciosa ou neoplásica), o prognóstico já se poderá agravar. No caso particular dos aneurismas saculares (a etiologia mais grave), a sua rutura pode levar a uma hemorragia subaracnoideia maciça, com complicações neurológicas severas e até a morte. ${ }^{1-2}$

Pretende-se com a descrição deste caso alertar para a importância do médico de família na continuidade e articulação de cuidados, atuando como provedor do utente. É primordial o seu papel na gestão da pluripatologia, o que por vezes evidencia a excessiva burocratização e complexidade do sistema de saúde. Este caso clínico é também importante do ponto de vista pedagógico para a prática clínica em cuidados de saúde 
primários, pois chama a atenção para uma complicação possível da diabetes: a paresia do III par craniano, que exige uma pronta atuação perante a sua suspeita.

\section{DESCRIÇÃO DO CASO}

Senhora de 55 anos, solteira, $12^{\circ}$ ano de escolaridade, reformada por invalidez (escriturária de contabilidade), da classe social média de Graffar. Reside com o pai, do qual é vítima de abuso psicológico, pertencendo a uma família com um Apgar de 3 (com disfunção acentuada). Mantém também uma relação conturbada com a mãe. Sem irmãos.

Embora apresente bom estado geral, trata-se de uma diabética tipo 1, de difícil controlo metabólico (HbAlc: $10 \%$ a $27 / 06 / 2019$, com valores $10-12 \%$ pelo menos desde 14/02/2013), com diagnóstico desde os sete anos de idade, já com complicações microvasculares (retinopatia com hemorragia vítrea - seguida em consulta de oftalmologia no hospital de referência) e macrovasculares (ateromatose carotídea assintomática); AVC sem sequelas e enfarte agudo do miocárdio com supradesnivelamento ST, condicionando insuficiência cardíaca com FEVE diminuída, com necessidade de implantação de CDI. A utente não possuía previamente uma equipa de saúde familiar atribuída e também havia deixado de ser seguida em consulta de endocrinologia, tendo sido por isso novamente referenciada. De entre a extensa lista de problemas que apresenta destacamse os seguintes antecedentes patológicos (Tabela 1):

Atualmente encontra-se medicada com insulina glargina 26U id (noite) e insulina lispro 3U 3id (e mais $1 \mathrm{U}$ por cada incremento de $50 \mathrm{mg} / \mathrm{dL}$ de glicemia se esta $>150 \mathrm{mg} / \mathrm{dL}$ ). Apresenta ainda como medicação crónica: ácido acetilsalicílico $100 \mathrm{mg}$ id, atorvastatina $40 \mathrm{mg}$ id, carvedilol 6,25mg 2id, pantoprazol 20mg id, levotiroxina $0,112 \mathrm{mg}$ id, levetiracetam $500 \mathrm{mg} 2 \mathrm{id}$, diazepam $5 \mathrm{mg} 2 \mathrm{id}$, quetiapina $100 \mathrm{mg}$ id e venlafaxina $150 \mathrm{mg}$ id.

A 12/05/2019, e em contexto de síndroma gripal, refere início súbito de dor ocular e supraciliar à direita, com os seguintes sintomas associados, também a nível do olho direito, visão turva, diplopia e fotofobia. Nesse mesmo dia dirigiu-se a um serviço de urgência convencionado, tendo tido alta medicada apenas para o quadro infecioso com paracetamol 500mg + cafeína $65 \mathrm{mg}$ 3id SOS e levocetirizina 5mg 2id, 10 dias. Embo- ra já resolvida a clínica respiratória, por não apresentar melhoria das queixas oculares, a 14/05/2019 recorreu a consulta de intersubstituição na sua UCSP e teve alta com a recomendação de aplicar dexametasona $1 \mathrm{mg} / \mathrm{mL}$ + neomicina $5 \mathrm{mg} / \mathrm{dL}$ colírio 2 gotas 3id e tomar paracetamol 1000mg 2id SOS. Por manutenção das queixas oftalmológicas, a 16/05/2019 (ou seja, quatro dias após o seu início) foi atendida em consulta aberta pela sua médica de família. Nesse dia, ao exame objetivo evidenciava ptose (bordo palpebral superior sobre o eixo visual) e exotropia do olho direito de novo na posição primária do olhar, com restrição nos movimentos oculares de adução e infraversão/supraversão. À inspeção não evidenciava outras alterações, nomeadamente secreções purulentas nem olho vermelho, e o olho esquerdo apresentava-se normal. Relativamente aos reflexos pupilares apresentava pupilas pouco reativas, em miose fixa bilateralmente, com a pupila direita irregular. Negava traumatismo crânio-encefálico, outros sinais focais de novo ou alteração de consciência. Foi então referenciada ao serviço de urgência, onde foi observada pelas especialidades de oftalmologia e neurologia. Do exame neuroftalmológico aí efetuado há ainda a destacar a ausência de defeitos de campo periférico por ameaça e de inatenção visual; a acuidade visual bilateral sem correção de $8 / 10$, com lente intraocular bilateral na câmara posterior e na fundoscopia marcas de laserterapia no fundo ocular bilateralmente, sem evidência de neovasos ativos, incluindo ausência de rubeose da íris. Ao exame neurológico exibia também prova de Barré com flexão e queda à direita, hemihipostesia álgica direita e défice motor distal na mão direita (antigos e já conhecidos), bem como hipostesia flutuante (por vezes à esquerda, por vezes à direita, no mesmo território).

A angioTC-CE efetuada no serviço de urgência revelou: "ausência de lesões endocranianas de natureza expansiva com tradução densitométrica. Identifica-se área de ténue hipodensidade e de Iimites mal definidos paraventricular frontal à direita, junto à transição corticomedular, de significado inespecífico, podendo ser vascular, sugerindo-se avaliação complementar por RM para melhor esclarecimento. Algumas imagens lacunares tálamo-capsulares à direita, vasculares ou espaços perivasculares alargados, igualmente melhor caracterizados por RM. Ateromatose calcificada nos sifões 
carotídeos, significativa para o grupo etário. Sem desvios da linha média, sem conflito no foramen occipital. Sistema ventricular adequado. Acentuação difusa dos sulcos, sem predomínio focal ou lobar. O estudo angioTC-CE assim efetuado não revelou anomalias vasculares nos planos obtidos, designadamente aneurismáticas ou vasculares malformativas e em particular afetando a porção posterior do polígono de Willis. Emergência fetal da artéria cerebral posterior esquerda, variante anatómica". A angioRM cerebral não foi realizada.

Tendo em conta a apresentação clínica, os seus antecedentes de diabetes e os achados da angioTC-CE (que permitiram excluir eventuais causas de compressão extrínseca do nervo oculomotor, como neoplasias, aneurismas ou anomalias vasculares malformativas) foi-lhe diagnosticada paresia do nervo oculomotor de etiologia microvascular, tendo tido alta para o seu médico assistente e ficando com consultas de oftalmologia e neurologia agendadas para cerca de um mês depois ( 26 e $27 / 06 / 2019$, respetivamente), onde já era seguida previamente.

Em consulta programada com a médica de família a 21/05/2019 apresentava ainda quadro clínico praticamente sobreponível, como sucedeu nas consultas de reavaliação de oftalmologia e neurologia. Contudo, a 10/07/2019, novamente em consulta com a médica de família, apresentava já alguma melhoria das queixas, especialmente da exotropia e diplopia, sendo que a remissão espontânea se verificou oito semanas após início do quadro.

Após a ocorrência da paresia, e até ida a consulta de endocrinologia, foi intensificado o regime insulínico, com aumento das unidades de insulina glargina de $26 \mathrm{U}$ para 30U à noite, e reforçada a necessidade de adesão ao esquema de insulina rápida. Embora a doente não seja hipertensa, foi solicitada também a automedição da pressão arterial, que revelou valores médios normais diurnos: 128/84.

\section{COMENTÁRIO}

A complexidade clínica de alguns doentes, ainda que relativamente jovens, constitui um verdadeiro desafio na prática clínica em medicina geral e familiar, como este caso retrata, pela necessidade de gestão de sérios e inúmeros de problemas de saúde. Revela-se não só um desafio em termos técnicos, bem como exige uma gestão eficiente da nossa agenda e da do utente. Registou-se, por exemplo, a necessidade de ao longo da prestação de cuidados referenciar a utente a diversas consultas hospitalares, algumas das quais onde era previamente seguida, mas por lacunas organizacionais dos diferentes serviços acabou por perder seguimento (como é o caso da sua diabetes), com prejuízo para a utente e contribuindo em muito para a sobrecarga burocrática sentida no dia-a-dia pelo médico de família. Contudo, cabe ao médico de família ser o coordenador dos cuidados a prestar, interagindo com as restantes especialidades, no superior interesse do utente. Além disso, embora a diabetes mellitus tipo 1 seja tradicionalmente de seguimento hospitalar, o médico de família é o médico assistente por excelência do seu utente, devendo conhecê-lo profundamente e acompanhá-lo no seu percurso de vida, vigiando e zelando pelo seu estado de saúde.

Por outro lado, a doente não tem apoios de uma rede familiar de apoio para lidar com os seus graves problemas de saúde, recusando para já uma eventual ajuda institucional.

Este caso evidencia ainda o facto de nem sempre as manifestações clínicas ocorrerem na realidade tal como se encontram descritas na teoria: esta doente não apresentava midríase (embora a pupila afetada se encontrasse irregular), o que indiciaria uma paresia incompleta do nervo oculomotor (de causa isquémica, como foi assumido). Porém, o reflexo pupilar encontrava-se alterado, o que já apontaria para uma eventual paresia total do nervo.

O facto da hipertensão e a diabetes serem as principais etiologias da paresia do III par craniano reforça a tónica da importância do controlo dos fatores de risco cardiovasculares, numa lógica de prevenção primária e secundária - como acontece neste caso. Numa tentativa de melhorar o controlo dos fatores de risco cardiovasculares foi intensificado o regime insulínico e a aumentada a vigilância da tensão arterial.

Por fim, a paresia do nervo oculomotor é uma manifestação pouco frequente de neuropatia diabética, mas com que devemos estar familiarizados, uma vez que os cuidados de saúde primários podem (e devem) ser o primeiro contacto e a entrada no sistema de saúde. Acarreta consigo elevado prejuízo funcional e 
estético para o doente, mas felizmente é uma condição potencialmente reversível. Contudo, pode ser o indício de situações potencialmente fatais, como seja um aneurisma sacular, o que acentua a importância do elevado grau de suspeição clínica e pronta atuação e orientação na presença de sinais de alarme de novo, como ptose, infraducção e abdução ocular, pupila fixa e midriática, diplopia e dor ocular.

\section{REFERÊNCIAS BIBLIOGRÁFICAS}

1. Goodwin J. Third nerve palsy (oculomotor nerve palsy). Medscape [Internet]; 2018 Oct 8 [cited 2019 Oct 1]. Available from: https:// emedicine.medscape.com/article/1198462-overview\#showall

2. Halvorson KL. Cranial nerve III palsy. In: Rosenfield M, Lee EM, Goodwin D, editors. Clinical Cases in Eye Care. Philadelphia:Wolters Kluwer; 2019. p. 453-6.
3. Gomes IR, Seldon R, Varandas G, Vieira ML. Paralisia do III par craniano com regeneração aberrante. Oftalmologia Rev Soc Port Oftalmol. 2016;40(4):335-8.

4. Pereira CU, Santos AC, Santos Jr JA, Campos GJ, Carvalho RW, Gusmão LC. Nervo oculomotor: anatomia, fisiologia e clínica [Oculomotor nerve: anatomy, physiology and clinical features]. Rev Cir Traumatol BucoMaxilo-Fac. 2012;12(2):93-104. Portuguese

\section{CONFLITO DE INTERESSES}

A autora declara não ter quaisquer conflitos de interesse.

\author{
ENDEREÇO PARA CORRESPONDÊNCIA \\ Ana Martins Ferreira dos Santos \\ E-mail: amfsantos86@hotmail.com \\ https://orcid.org/0000-0001-6652-4200
}

Recebido em 29-10-2019

Aceite para publicação em 22-06-2020

\section{ABSTRACT \\ OCULOMOTOR NERVE PALSY: ONE ENTITY SOMETIMES DIFFICULT TO MANAGE IN GENERAL AND FAMILY MEDICINE}

Introduction: The oculomotor nerve palsy is rare in the general population, affecting mainly people over 60 years. It may course with eye pain, diplopia, ptosis, and strabismus. Hypertension and diabetes are its main etiologies. Mononeuropathies affect $3 \%$ of the diabetic population. It can be a manifestation of potentially fatal situations, such as cerebral aneurysms.

Case description: A 55-year-old woman in good general condition, although with several important comorbidities, including type 1 diabetes mellitus with poor metabolic control. On May $12^{\text {th }}$ of 2019 , she reports the sudden onset of right supraciliary and ocular pain, with the following associated symptoms, also affecting the right eye: blurred vision, diplopia, photophobia, and new divergent strabismus. After two previous contacts with health institutions for this reason, she was referred to the emergency department by her family doctor, four days after the onset of the complaints, and was diagnosed with third nerve palsy. It was adopted an expectant attitude, with the spontaneous remission of symptoms occurring eight weeks later.

Comments: The aim of this case is to warn of the importance of the family doctor in the continuity of health care because he/she is the assistant physician par excellence of his/her patient, will be the one who knows him best, and can easily value important new changes that may arise. On the other hand, it aims to emphasize the importance of quick action in the case of clinical suspicion of third nerve palsy, as well as the control of cardiovascular risk factors in a diabetic population.

Keywords: Oculomotor nerve disease; Diabetes mellitus. 